University of South Florida

DIGITAL COMMONS

Digital Commons @ University of

@ UNIVERSITY OF SOUTH FLORIDA

South Florida

$3-2006$

\title{
Observing seasonal Steric Sea Level Variations with GRACE and Satellite Altimetry
}

Don P. Chambers

University of South Florida, donc@usf.edu

Follow this and additional works at: https://digitalcommons.usf.edu/msc_facpub

Part of the Marine Biology Commons

\section{Scholar Commons Citation}

Chambers, Don P., "Observing seasonal Steric Sea Level Variations with GRACE and Satellite Altimetry" (2006). Marine Science Faculty Publications. 182.

https://digitalcommons.usf.edu/msc_facpub/182

This Article is brought to you for free and open access by the College of Marine Science at Digital Commons @ University of South Florida. It has been accepted for inclusion in Marine Science Faculty Publications by an authorized administrator of Digital Commons @ University of South Florida. For more information, please contact digitalcommons@usf.edu. 


\title{
Observing seasonal steric sea level variations with GRACE and satellite altimetry
}

\author{
Don P. Chambers ${ }^{1}$ \\ Received 9 February 2005; revised 28 June 2005; accepted 7 December 2005; published 21 March 2006.
}

[1] Sea level rises and falls as the temperature and salinity of the water column varies, which is known as steric sea level. Sea level also changes as water mass is redistributed within the ocean or is added or removed. Satellite radar altimeters measure the combination of both effects, while the Gravity Recovery and Climate Experiment (GRACE) was designed to measure time variable gravity caused by movement of water mass. Theoretically, altimetry and GRACE data can be combined in order to compute the steric sea level variations. We test this by combining current GRACE and Jason 1 altimeter data and comparing against steric sea level observations. We will describe how to properly combine the altimetry and GRACE data, commenting on important corrections that need to be applied to each data type. Using empirical orthogonal function (EOF) analysis, we examine the leading modes of seasonal variability and find that using GRACE improves the ability to recover the dominant mode of steric sea level variability over using altimetry alone. The RMS error of the GRACE ocean mass variations is estimated to be about $2 \mathrm{~cm}$ of sea level at a $1000 \mathrm{~km}$ smoothing radius. Although this is larger than initially predicted from the GRACE mission, it is still significantly smaller than the recovered signal in several regions of the ocean.

Citation: Chambers, D. P. (2006), Observing seasonal steric sea level variations with GRACE and satellite altimetry, J. Geophys. Res., 111, C03010, doi:10.1029/2005JC002914.

\section{Introduction}

[2] There are two major components of sea level variability. Changes in the temperature and salinity cause the water to expand or contract, which is known as the steric component. These fluctuations do not cause changes in the ocean bottom pressure, since the density compensates to counteract the sea level change. Other sea level variations do cause a change in ocean bottom pressure, however. The source of these variations is either ocean mass redistribution (such as caused by tides or changes in the ocean circulation) or water mass flux (such as the global water cycle or melting of glaciers and ice sheets).

[3] Satellite altimetry measures the combined effect of these steric and mass variations. Regular, continuous altimeter measurements of sea level have been made since 1992 at 10-day intervals by the TOPEX/Poseidon and Jason 1 missions. The spatial sampling is approximately $300 \mathrm{~km}$ west to east at the equator (because of the ground track spacing), and less than $7 \mathrm{~km}$ along the ground track. Although temperature and salinity have been measured by profilers and buoys for decades and converted to steric sea level (SSL) variations, the spatial and time resolution is not nearly as good as that for altimetry. Because of this, it is

\footnotetext{
${ }^{1}$ Center for Space Research, University of Texas at Austin, Austin, Texas, USA.

Copyright 2006 by the American Geophysical Union. 0148-0227/06/2005JC002914
}

nearly impossible to compute SSL at regular, monthly periods except at some locations. One example is in the equatorial Pacific, where the Tropical Atmosphere-Ocean (TAO) moored buoys have made hourly observations at fixed locations since the early 1990s [e.g., Menkes et al., 1995]. Global analysis of subsurface temperature and salinity data have typically been done by computing monthly means using decades of data, so represent only a mean climatology [e.g., Stephens et al., 2002]. Analysis of interannual temperature fluctuations (and hence SSL) has been done, but only for 1- to 5-year averages [Levitus et al., 2000; Willis et al., 2004].

[4] Until recently, the mass component could only be inferred from ocean models that do not include water mass flux between the ocean and land [e.g., Ponte, 1999] or from a limited number of ocean bottom pressure gauges [e.g., Hughes and Smithson, 1996]. Thus, although there have been regular, nearly global measurements of the total sea level variation since 1992 when the TOPEX/Poseidon and ERS-1 missions were launched, there have been only limited measurements of the individual components.

[5] This has changed recently with the launch of the Gravity Recovery and Climate Experiment (GRACE) in March 2002. The primary science goal of the GRACE mission is to determine variations in the Earth's gravity field at monthly intervals and at a spatial resolution of several hundred $\mathrm{km}$. Theoretically, the dominant source of time variable gravity on periods of less than several hundred years is movement of water mass. On the basis of this, Wahr et al. [1998] derived a methodology to convert the GRACE 
observations into surface mass variations, while Jayne et al. [2003] expanded the procedure to describe how to combine satellite altimetry and GRACE data to estimate SSL (and heat storage) variations. Because there were no GRACE observations available at the time of their study, Jayne et al. [2003] simulated measurements based on ocean bottom pressure from a model as well as output from a hydrology model in order to quantify the effect of using GRACE with altimetry opposed to altimetry alone to recover heat storage. They found noticeable improvements, generally poleward of $30^{\circ}$.

[6] Gravity field coefficients have been produced by the GRACE project nearly monthly since August 2002. Although several papers have been published examining the GRACE results over the land, only a few have examined the results over the ocean. Wahr et al. [2004] compared sea level maps derived from the GRACE gravity field estimates with maps determined from a combination of hydrology and ocean models. They found significant differences at the 2-3 cm level. Chambers et al. [2004] examined the global average of the ocean signal and found that GRACE clearly observed the seasonal exchange of water mass between the ocean and land, which has an amplitude of 8-9 $\mathrm{mm}$. Theoretically, the ocean sea level should readjust to water mass fluxes via barotropic waves and distribute the signal more or less uniformly over the oceans within a few days. At least some of the differences between GRACE and the model used by Wahr et al. [2004] can be attributed to this global mass exchange, which was not accounted for in the model.

[7] In this investigation, we will look at the GRACE data over the ocean by combining it with data from the Jason 1 altimeter mission and comparing it with seasonal SSL computed from the World Ocean Atlas 2001 (WOA01) database [Stephens et al., 2002]. As mentioned earlier, this is one of the few analyses of temperature/salinity data on both a global spatial scale and a monthly timescale. Other global analyses [Levitus et al., 2000; Willis et al., 2004] are limited to multiyear averages. One important difference in this analysis is that it does not ignore the exchange of water mass between the land and ocean that is contributing to the GRACE signal. It has been demonstrated that satellite altimeters also detect the water mass exchange over the ocean if the data are corrected for SSL variations [Chen et al., 1998; Minster et al., 1999]. One limitation of this study is that the steric signal we will be using for validation is only a mean monthly climatology; that is, it only represents a mean signal for a month (i.e., the average computed from 70 or more Aprils), while the Jason 1 and GRACE data will be from a particular month (i.e., April 2003).

[8] We will compare SSL computed from WOA01 with an estimated SSL (ESSL) determined from a combination of altimetry and GRACE data. If the GRACE data are observing real signals over the ocean, then a combination of GRACE and altimetry should provide an ESSL that is closer to SSL than using altimetry alone, similar to what Jayne et al. [2003] found for their simulations of heat storage. We will focus on the seasonal cycle because this is expected to be the dominant signal observable with the two years of data available from GRACE and because of the limitation in the SSL data. We will use empirical orthogonal function (EOF) Analysis, also know as Principal Compo- nent Analysis [Preisendorfer, 1988], to extract the seasonal variation. EOFs can be determined from a time series of gridded data (e.g., $h(\phi, \lambda, t))$ and separate the signal into a spatial pattern (the EOF mode) and a time series (the principal component) representing the time variation of the spatial pattern so that

$$
h(\phi, \lambda, t)=\sum_{k=1}^{N} \alpha_{k}(\phi, \lambda) \beta_{k}(t)
$$

where $\phi$ is the latitude of the grid, $\lambda$ is the longitude, $t$ is time, $k$ is the EOF mode, $N$ is the maximum number of modes (equivalent to the number of unique time points), $\alpha_{k}$ is the $k$ th EOF mode and $\beta_{k}$ is the associated principal component. Using EOF analysis can show more detail in the seasonal cycle opposed to harmonic analysis at the annual frequency, since the variability may not be exactly sinusoidal at a yearly frequency and because there may be more than one mode of annual variability, out of phase with each other. Because EOF analysis does not assume either a pattern or frequency of variability, it can better detect changes in the frequency, as well as modes with different phases but similar frequencies.

[9] In the following section, we will describe in detail how the GRACE gravity coefficients are converted into sea level variations, as well as how altimeter and SSL variations are smoothed to be comparable to the GRACE maps. In addition, we will discuss several important corrections that must be made to the GRACE data in order to reconcile them with the altimeter measurements, before combining. Several of these corrections have already been noted by both Chambers et al. [2004] and Wahr et al. [2004]. After discussing the processing, we will compare the leading EOF modes and discuss the results.

\section{Data Processing}

\subsection{Inferring Sea Level Variations From GRACE Gravity Coefficients}

[10] GRACE does not measure variations in gravity or mass directly, but instead measures changes in range between the two GRACE spacecraft. In geodesy, the Earth's gravity field potential $(V)$ at any point exterior to the Earth system is typically expressed as an infinite spherical harmonic series that is a function of the Earth's gravitational constant $(\mu)$, the mean equatorial radius $\left(a_{E}\right)$, the geocentric radius to the point $(R)$, and the geographic latitude $(\phi)$ and longitude $(\lambda)$ [e.g., Heiskanen and Moritz, 1967]

$$
\begin{aligned}
V(R, \phi, \lambda, t)= & \frac{\mu}{R}+\frac{\mu}{R} \sum_{l=1}^{N \max }\left(\frac{a_{E}}{R}\right)^{l} \sum_{m=0}^{l} \\
& \cdot P_{l m}(\sin \phi)\left\{C_{l m}(t) \cos m \lambda+S_{l m}(t) \cos m \lambda\right\}
\end{aligned}
$$

where $P_{l m}(\sin \phi)$ are the fully normalized Associated Legendre Polynomials of degree $l$ and order $m . C_{l m}$ and $S_{I m}$ are the fully normalized spherical harmonic geopotential coefficients. Here we have assumed that the geopotential coefficients can vary in time, although in many applications they are treated as time invariant. Although theoretically the series is exact only if the summation is carried out to an 


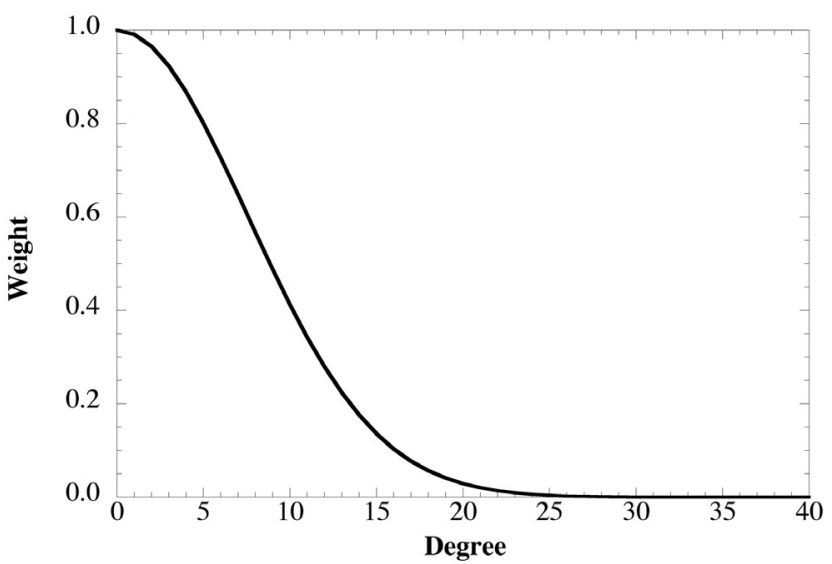

Figure 1. Weighting function used to smooth GRACE gravity coefficients.

infinite degree, in practice it is truncated at some value Nmax. The normalization factor used by the GRACE project is described by Bettadpur [2004].

[11] The GRACE project then uses the observed variations in the range rate between the two satellites and other tracking data to estimate the values of the gravitational coefficients along with other dynamical orbit parameters in a least squares estimation in order to minimize the misfit between a modeled orbit (based on the gravitational potential) and the observations [Bettadpur, 2004]. Estimations of the gravity field coefficients are made approximately every month to degree/order 120, which corresponds to wavelengths $\sim 300 \mathrm{~km}$ and longer (wavelength $\sim 40000 \mathrm{~km} / \mathrm{L}$ ).

[12] The month gravity field coefficients can be converted to time-varying gravity coefficients $\left(\Delta C_{l, m}(t)\right.$, $\left.\Delta S_{l, m}(t)\right)$ by computing and removing the time mean. Wahr et al. [1998] detailed the methodology for converting time variable gravity field coefficients into maps of surface mass density on the basis of the assumption that for periods less than several hundred years the primary cause of temporal changes in the Earth's gravity field is movement of water mass within the Earth's relatively thin fluid envelope

$$
\begin{aligned}
\Delta \sigma(\phi, \lambda, t)= & \frac{a_{E} \rho_{E}}{3} \sum_{l=0}^{120} \sum_{m=0}^{l} \frac{(2 l+1)}{\left(1+k_{l}\right)} P_{l m}(\sin \phi) \\
& \cdot\left\{\Delta C_{l m}(t) \cos m \lambda+\Delta S_{l m}(t) \cos m \lambda\right\}
\end{aligned}
$$

where $\Delta \sigma$ is the surface mass density in $\mathrm{kg} \mathrm{m}^{-2}, \rho_{E}$ is the average density of the Earth $\left(5517 \mathrm{~kg} \mathrm{~m}^{-3}\right)$, and $k_{l}$ are load Love numbers of degree $l$. Surface mass density is not a meaningful quantity for oceanographic applications, but it is straightforward to convert it to either anomalies of ocean bottom pressure or equivalent surface elevation [Jayne et al., 2003]. The latter quantity is most useful for this study, as this is equivalent to the sea level change caused by barotropic and ocean mass variations; i.e., the nonsteric sea level. It is simply $\left(\Delta \sigma / \rho_{W}\right)$, where $\rho_{W}$ is the density of fresh water $\left(1000 \mathrm{~kg} \mathrm{~m}^{-3}\right)$. In this paper, we will refer to such sea level variations as $\Delta \eta_{\text {mass }}$.
[13] If the GRACE coefficients had no error, or very low error compared to the signal, one could use equation (3) to produce maps of $\Delta \eta_{\text {mass }}$ representing the average mass density for a radius of about $150 \mathrm{~km}$ (half the wavelength). However, the errors are significantly larger than the expected time variable signal except at the longest wavelengths [e.g., Wahr et al., 2004, Figure 1]. To reduce the impact of the error at increasing degree (smaller wavelength), one can average over a larger radius. Wahr et al. [1998] demonstrated a method to compute spatially averaged maps directly from the coefficients, using an averaging function that is a function of degree $\left(W_{l}\right)$. The averaging function was derived from a spatial Gaussian function. The half-power point of the Gaussian is determined by a parameter referred to as the averaging radius, $r$. The filter suppresses signals with a wavelength shorter than $2 r$, provided that the error characteristics are random. Wahr et al. [1998] presented a recursive algorithm to derive the averaging function given $r$, but we found instabilities beyond $\sim$ degree 50 . Instead, we use an analytical function that approximates the function of Wahr et al. [1998]

$$
W_{l}=\exp \left[-\frac{\left(l r / a_{E}\right)^{2}}{4 \ln (2)}\right]
$$

[14] Figure 1 shows the value of $W_{l}$ as a function of degree with $r=1000 \mathrm{~km}$. Once the averaging function has been determined, one can modify equation (3) to compute smoothed maps of $\left(\Delta \eta_{\text {mass }}\right)$

$$
\begin{aligned}
\Delta \eta_{\text {mass }}(\phi, \lambda, t)= & \frac{a_{E} \rho_{E}}{3 \rho_{W}} \sum_{l=0}^{120} \sum_{m=0}^{l} \frac{(2 l+1)}{\left(1+k_{l}\right)} W_{l} P_{l m}(\sin \phi) \\
& \cdot\left\{\Delta C_{l m}(t) \cos m \lambda+\Delta S_{l m}(t) \cos m \lambda\right\} .
\end{aligned}
$$

[15] Wahr et al. [2004] demonstrated the effect of different averaging radii on the GRACE data. They concluded that at a smoothing radius of $1000 \mathrm{~km}$ the GRACE annual amplitude has an error of $1 \mathrm{~cm}$ at most. We choose this smoothing radius for our investigation, and use the Release-01 gravity coefficients distributed by the GRACE project computed at the Center for Space Research for August-November 2002, February-May 2003, and July 2003 to July 2004 [Bettadpur, 2004]. No coefficients are currently available for the missing time periods.

[16] Because the averaging function is so wide, there is a problem in ocean areas within $\sim 1000 \mathrm{~km}$ of land with large hydrology signals. For instance, the hydrology variation over the Amazon basin is tens of $\mathrm{cm}$ of water, while the expected ocean variations are a few $\mathrm{cm}$ at most. Because the smoothing is done on global spherical harmonics, any large variation over land will contribute to the average over the ocean within the smoothing radius. To mitigate this problem in our analysis, we mask all the grids on land and all ocean grids within $1000 \mathrm{~km}$ of coastlines. We do not mask around small islands.

[17] The GRACE satellites orbit approximately $400 \mathrm{~km}$ above the Earth's surface, which means that a portion of the gravitational signal they detect is due to mass variation within the atmosphere below them. This means that if 
atmospheric mass variations were not modeled, then the maps from equation (5) would also contain the effect of the atmospheric mass change. However, the GRACE project models the gravity variations in the atmosphere below GRACE altitude on the basis of pressures from the European Center for Medium-Range Weather Forecasting (ECMWF) model, as well as barotropic sea level from a model forced by ECMWF winds and pressures [Flechtner, 2003]. Thus the gravity coefficients provided by the GRACE project have already had a model of atmospheric mass removed, as well as a barotropic model. The implications of modeling a barotropic ocean component will be discussed further in section 2.4.2. Any errors in modeling the atmosphere will contribute to errors in the interpretation of GRACE data as mass variations over the ocean or land. Although the estimate of the atmospheric mass is obviously not exact, it is arguably the best available.

\subsection{Jason 1 Altimetry}

[18] We use sea surface height (SSH) data from the Jason 1 altimeter for August 2002 to July 2004. All standard geophysical and atmospheric corrections are applied, including removing ocean tides and an inverted barometer correction. We remove a global bias of $15 \mathrm{~cm}$ to align the Jason 1 data with the TOPEX data and compute mean SSH profiles and cross-track gradients for each ground track pass on the basis of data from both satellites over an 11-year period [Chambers, 2002]. We interpolate data to the mean profile on the basis of the estimated gradients and compute $\mathrm{SSH}$ anomalies relative to the mean profiles at approximately a $6 \mathrm{~km}$ resolution along the ground track. The mean of the anomalies for August 2002 to July 2004 is then removed to readjust the data to match with the GRACE mean.

[19] An additional correction has also been applied to account for two changes in the behavior of the Jason 1 Microwave Radiometer (JMR) over the lifetime of the mission. The JMR is used to correct the altimeter range for path delay caused by water vapor in the troposphere. At the first Ocean Surface Topography Science Team Meeting [Fu, 2004], JMR engineers reported two significant anomalies in the instrument. In November 2002, the path delay correction became biased by about 3-4 $\mathrm{mm}$. In November 2003, it became biased again by a further $7-8 \mathrm{~mm}$ in the same direction. The cause is theorized to be thermal shock to the instrument, and the error will bias the SSH measurement globally by the same amount as the path delay bias. Although the Jason 1 data are currently being reprocessed to correct for this, none of the data have been released. Because this error accounts to a step function of more than $1 \mathrm{~cm}$ over the two years of SSH data being studied, and this is the nearly at the level of the signal we are expecting to observe, we apply an ad hoc correction to remove the two jumps. The correction is based on the mean difference between the path delay determined from the JMR and that computed from the ECMWF atmosphere model. The mean difference between the two was very small before November 2002 and there have been no reported changes to the model, so it is assumed the differences track the JMR bias changes.
[20] The along-track SSH anomalies (SSHAs) are then mapped to a $1^{\circ}$ grid using weighted averages where the weights $\left(W_{S}\right)$ are of the form

$$
W_{S}=\exp \left[-4\left(\frac{r}{R_{S}}\right)^{2}\right]
$$

and $r$ is the distance (in $\mathrm{km}$ ) from the center of the grid for which the average is desired to the $n$th grid around it and $R_{S}=500 \mathrm{~km}$. Data within a radius of $500 \mathrm{~km}$ were used to compute the average. This was done to completely fill in grids over the ocean before continuing with the next step.

[21] Since the GRACE results were mapped from weighted spherical harmonic coefficients, we have attempted to compute smoothed maps of altimeter SL in as similar as way as possible. First, we compute spherical harmonic coefficients from the gridded data $(\operatorname{SSHA}(\phi, \lambda, \mathrm{t}))$, after setting land and remaining ocean regions with no data (e.g., poleward of $66^{\circ}$ latitude) to zero and then integrating over the entire spherical area of the Earth $(\Omega)$

$$
\begin{aligned}
\Delta \hat{C}_{l m}(t) & =\int_{\Omega} \operatorname{SSHA}(\phi, \lambda, t) P_{l m}(\sin \phi) \cos m \lambda d \Omega \\
\Delta \hat{S}_{l m}(t) & =\int_{\Omega} \operatorname{SSHA}(\phi, \lambda, t) P_{l m}(\sin \phi) \sin m \lambda d \Omega
\end{aligned},
$$

where the $\left(^{\wedge}\right)$ symbol reminds the reader that these coefficients are not the same as the dimensionless gravity coefficients from GRACE, but have units the same as SSHA. The coefficients are computed for all degrees and orders from 0 to 120 , since the bias term $\Delta C_{00}$ is important here in order to completely reproduce the input grid. The Associated Legendre Polynomials are the same fully normalized ones used for the GRACE processing. Using these coefficients, the smoothed sea level maps from altimetry can then be computed using the same averaging function as GRACE (equation (4))

$$
\begin{aligned}
\Delta \eta_{\text {alt }}(\phi, \lambda, t)= & \frac{1}{4 \pi} \sum_{l=0}^{120} \sum_{m=0}^{l} W_{l} P_{l m}(\sin \phi) \\
& \cdot\left\{\Delta \hat{C}_{l m}(t) \cos m \lambda+\Delta \hat{S}_{l m}(t) \cos m \lambda\right\}
\end{aligned}
$$

The factor of $1 / 4 \pi$ is included to account for the spherical area of the Earth that was introduced in the formulation in equation (7). The smoothed grid is evaluated at $1^{\circ}$ intervals and land and ocean areas within $1000 \mathrm{~km}$ of land are masked as with the GRACE maps.

[22] Obviously, spherical harmonic coefficients from a gridded data set (that has already been smoothed to a certain extent) are not identical to the ones computed using a statistical estimation technique from the raw data such as with GRACE. However, this methodology is arguably better than applying a filter that attempts to duplicate equation (4) in the space domain. At least the smoothing in both cases has been done on the coefficients using the same averaging function.

\subsection{Steric Sea Level}

[23] The SSL anomalies are computed from the most recent World Ocean Atlas 2001 (WOA01) database [Stephens 
et al., 2002]. The steric variation for each $1^{\circ}$ grid point and month is computed by converting the mapped temperature and salinity values into density $(\rho)$ and integrating over the water column

$$
\Delta \eta_{S S L}(\phi, \lambda, t)=\frac{1}{\rho_{0}} \int_{-h}^{\eta}[\rho(\phi, \lambda, t, z, T, S)-\bar{\rho}(\phi, \lambda, z, \bar{T}, \bar{S})] d z
$$

where $\eta$ is the sea surface, $h$ is the maximum depth, $\rho_{0}$ is the reference density $\left(1027 \mathrm{~kg} \mathrm{~m}^{-3}\right)$, and the overbar indicates an average (computed from the mapped long-term mean temperature and salinity grids in WOA01). The maximum depth in the monthly WOA01 fields is $1500 \mathrm{~m}$, so the integration was carried to this level. The temperature and salinity at lower levels were assumed not to change, which is a reasonable expectation for seasonal timescales. Note that the objective mapping used to create the WOA01 temperature and salinity data relied on the same weighting function we used in the initial altimeter maps (equation (6)), but as a three-step iteration starting with $R_{S}=888 \mathrm{~km}$ and ending with $R_{S}=444 \mathrm{~km}$. Thus the SSL anomalies we compute are smoothed to some extent, but not in the same fashion as the GRACE and altimeter maps. To be as consistent as possible, we convert the SSL anomalies were to spherical harmonics and regrid them to a smoothed map analogous to the process described in section 2.2 for altimetry.

\subsection{Reconciling GRACE and Altimetry}

[24] The GRACE and Jason 1 projects use different standards in their processing. This results in several important discrepancies in background models. Here, we discuss the important issues that affect the combination of GRACE and Jason 1 data, and provide a roadmap for reconciling the two data sets.

\subsubsection{GRACE Coefficients With $l=0,1$}

[25] Note that equation (5) is formulated to use gravity coefficients down to degree 0 . In the Earth gravity field expansion, $C_{00}$ represents the total mass of the Earth (including solid Earth, oceans, atmosphere, etc.), and is constant. Because of this, $\Delta C_{00}$ from GRACE can be assumed to be zero at all times [Wahr et al., 1998].

[26] The terms with $l=1$ are proportional to the position of the Earth's geocenter relative to an Earth-fixed reference frame [e.g., Cretaux et al., 2002]. There are two ways to handle these terms in the gravity field coefficients. The first way is to reference the gravity field to the Earth's instantaneous geocenter, so that $\Delta C_{10}=\Delta C_{11}=\Delta S_{11}=0$ for all times. This is the convention adopted by the GRACE project. The second way is to reference the gravity field to a fixed reference frame, and allow the $l=1$ terms to be nonzero. The main cause of geocenter variations on seasonal timescales is water mass movement [e.g., Cretaux et al., 2002]. It has also been demonstrated that altimeter SL (when combined with SSL and hydrology estimates) can be used to estimate geocenter variations that agree favorably with other geocenter estimates [Chen et al., 1999]. Thus altimetry includes the ocean mass variations that cause geocenter motion, whereas the GRACE gravity coefficients will not because of the convention adopted by the project.

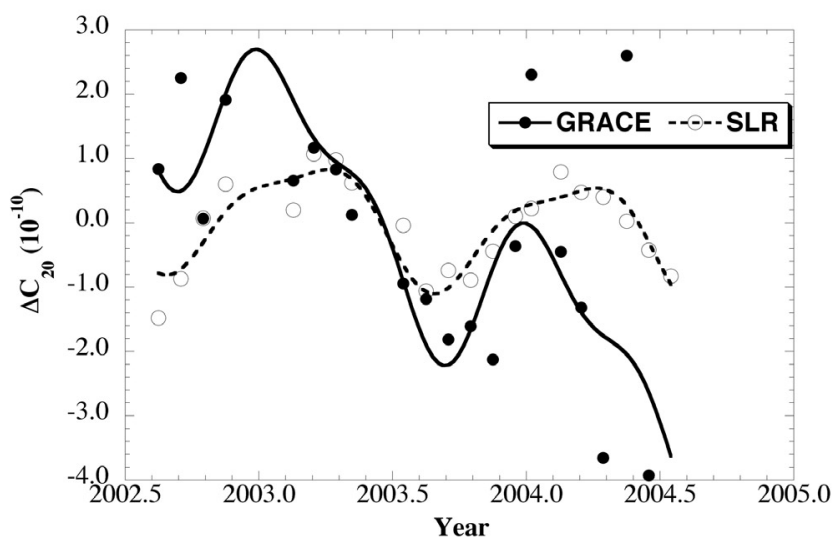

Figure 2. $\Delta C_{20}$ from GRACE and SLR analysis. An atmosphere $\Delta C_{20}$ from the GRACE AOD model has been removed from the SLR data to be consistent with GRACE.

[27] Chambers et al. [2004] demonstrated that adding a model of the degree 1 variations to the GRACE coefficients produced a better determination of the global seasonal ocean mass variation. The model was an annual sinusoid fit to geocenter estimates computed from satellite laser ranging (SLR) data [Chen et al., 1999]. Because we will be combining the GRACE data with altimetry, it is important to reconcile the data regarding the degree 1 terms. We find it conceptually more straightforward to convert geocenter variations $(\Delta x(t), \Delta y(t), \Delta z(t))$ to degree 1 gravity coefficient anomalies, since

$$
\begin{aligned}
\Delta C_{10}(t) & =\frac{\Delta z(t)}{a_{E} \sqrt{3}} \\
\Delta C_{11}(t) & =\frac{\Delta x(t)}{a_{E} \sqrt{3}} \\
\Delta S_{11}(t) & =\frac{\Delta y(t)}{a_{E} \sqrt{3}}
\end{aligned}
$$

Then one can use equation (5) starting at degree 1 to compute $\Delta \eta_{\text {mass }}$ using the coefficients calculated with equation (10). Also note that although the geocenter is a point relative to the reference axis center, the associated gravitational signal has a wavelength of $\sim 40000 \mathrm{~km}$, with an amplitude that is multiplied by a factor of approximately 3 to convert to equivalent water level (equation (5)). Since the sea level variation related to the geocenter motion is a juxtaposition of three long-wavelength signals, ignoring variations in geocenter with even a few $\mathrm{mm}$ amplitude can possibly cause errors of nearly $1 \mathrm{~cm}$ of sea level. So, although there are still disagreements in geocenter solutions at the few mm level, we believe the error in the solution is less than the error in ignoring it. We will examine this further in section 3.1, when we compare results using four different geocenter models and no geocenter model.

\subsubsection{Problems With $\Delta C_{20}$}

[28] Analysis of SLR tracking data to geodetic satellites has also proven to be a robust measure of the time variation in the $C_{20}$ gravity coefficient, which is caused by water mass transport [e.g., Chao and Eanes, 1995; Nerem et al., 2000; Cox and Chao, 2002; Cheng and Tapley, 2004]. 

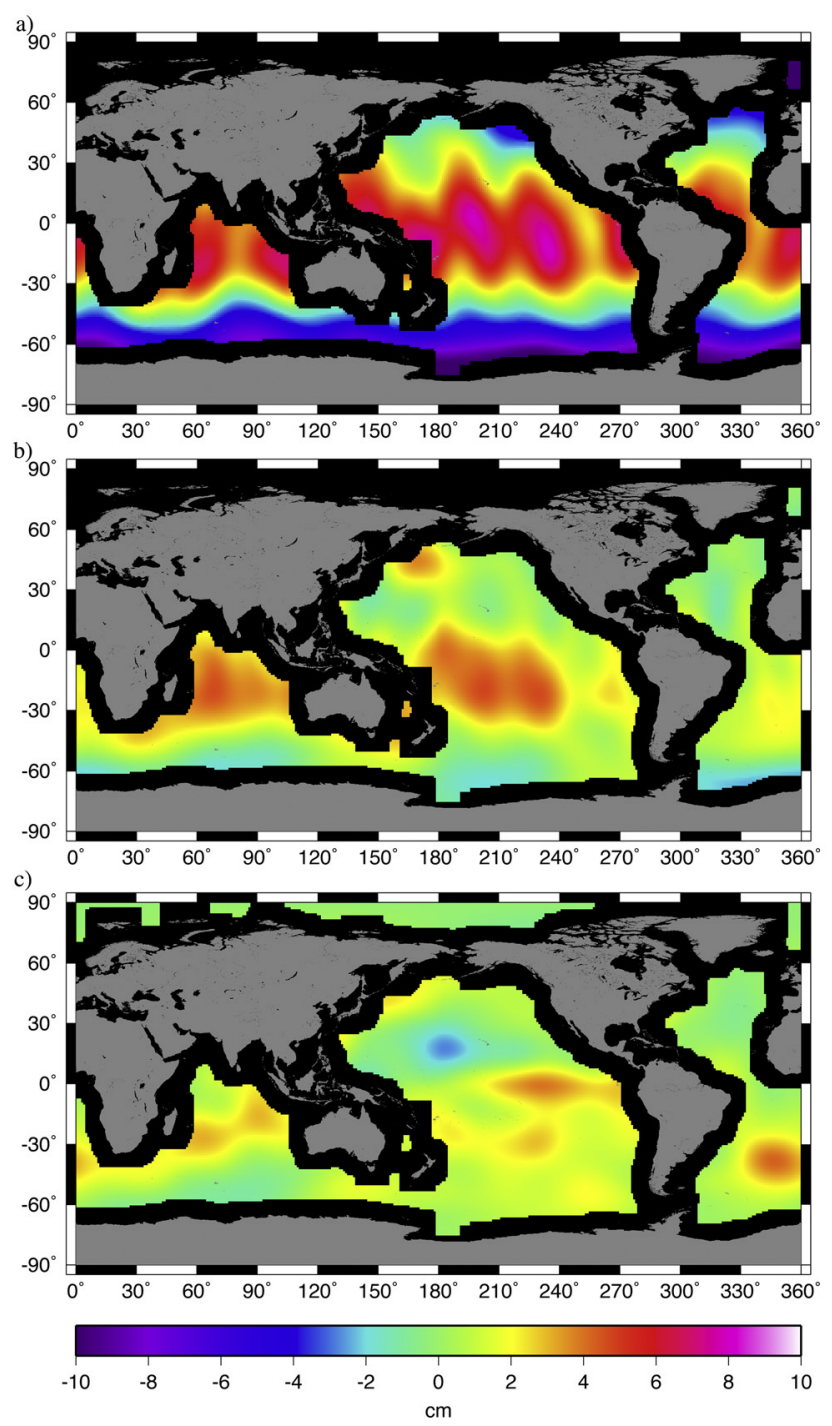

Figure 3. Leading EOF spatial maps of (a) GRACE, (b) GRACE with SLR $\Delta C_{20}$ substituted, and (c) Jason 1WOA01.

Figure 2 shows the time series of variations in $\Delta C_{20}$ measured by GRACE and that determined from an analysis of SLR tracking to geodetic satellites [Cheng and Tapley, 2004]. Although the GRACE $\Delta C_{20}$ estimates are close to those determined from the SLR analysis for part of 2003, there are significant differences in 2002 and 2004. The GRACE values in 2002 are several times more positive than those determined from the SLR analysis in 2002, while the values in 2004 are several times more negative. This results in an apparent trend in the GRACE values that is not present in the SLR values, as well as larger annual variations that are out of phase with the expected values.

[29] Figure 3a shows the leading EOF computed from the 21 months of GRACE maps using the values of $\Delta C_{20}$ from GRACE, while Figure $3 b$ shows the map where the SLR values have been substituted. Figure $3 c$ shows the pattern predicted from the difference of $\Delta \eta_{\text {alt }}$ and $\Delta \eta_{\mathrm{SSL}}$. Figure 4 shows the time series associated with the spatial maps shown in Figure 3. It is clear that using the GRACE $\Delta C_{20}$ values causes an unexpectedly large zonal variation in SL, of the order of tens of $\mathrm{cm}$ instead of a few $\mathrm{cm}$. On the other hand, using the values determined from an SLR analysis results in a pattern that is closer to the predicted one. Although there are still some differences between Figures $3 b$ and $3 c$, the correlation is significant at 0.7 .

[30] Some authors [e.g., Wahr et al., 2004] have ignored $\Delta \mathrm{C}_{20}$ in their calculations because of this problem. However, we know that a significant portion of $\Delta C_{20}$ is related to mass redistribution in the ocean [Cheng and Tapley, 2004]. Therefore we choose to substitute the SLR values into our analysis in place of the GRACE values instead of ignoring a portion of the ocean mass variation. Although this is obviously not the preferred way to fix this problem, we feel it is the best option until it is corrected in the processing stage. Recent experiments by the GRACE project appear to have resolved the problem, and reprocessed GRACE data should have significantly improved $\Delta C_{20}$ coefficients comparable to those from SLR analysis (S. Bettadpur, personal communication, 2005).

\subsubsection{Ocean Pole Tide}

[31] The ocean pole tide has not been modeled in the GRACE processing, as is routinely done in the altimetry processing. Thus the GRACE maps will contain the monthly SL variations associated with the pole tide, while the altimetry will not. The wavelength of the ocean pole tide signal is long $(\sim 20000 \mathrm{~km})$ and has an amplitude of nearly $1 \mathrm{~cm}$ with an annual period. If this is not removed from the GRACE maps it will cause a detectable difference at the level of the estimated error when combined with altimetry. To correct GRACE for this, we map the ocean pole tide variation on the basis of the IERS polar motion time series and the model used in the $\mathrm{T} / \mathrm{P}$ and Jason 1 altimeter processing [Wahr, 1985], averaged over the same time period as GRACE. These ocean pole tide maps are then subtracted from the GRACE maps to reconcile them with altimetry. We note that GRACE will be including a background ocean pole tide model in the next reprocessing so this will not be an issue in Release-02 data ( $\mathrm{S}$. Bettadpur, personal communication, 2005).

\subsubsection{Background Barotropic Model}

[32] As mentioned previously, GRACE processing also removes variability from an ocean barotropic model along

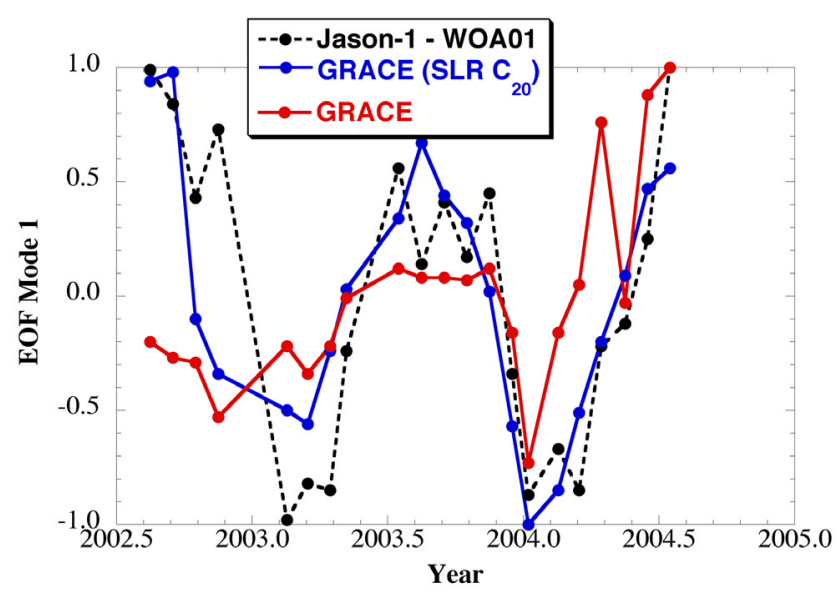

Figure 4. Associated principal components with leading EOF maps shown in Figure 3. 
Table 1. Geocenter Models Used in Analysis, Showing Data Used, Reference, and Annual Fits to Positions in mm of Amplitude $(A)$ and Phase $(\theta)$ in Degrees Using $A \cos (\omega t-\theta)$, Where $t=0$ is January 1

\begin{tabular}{cccccc}
\hline Model & Data Used & $\begin{array}{c}\Delta \mathrm{x}, \\
\mathrm{amp} / \mathrm{ph} \text { ase }\end{array}$ & $\begin{array}{c}\Delta \mathrm{y}, \\
\mathrm{amp} / \mathrm{ph} \text { ase }\end{array}$ & $\begin{array}{c}\Delta \mathrm{z}, \\
\text { amp/phase }\end{array}$ & \multicolumn{1}{c}{ Reference } \\
\hline 1 & SLR (Lageos 1 + 2) & $2.2 / 59^{\circ}$ & $3.2 / 299^{\circ}$ & $2.8 / 45^{\circ}$ & Chen et al. $[1999]$ \\
2 & SLR/DORIS (T/P) & $1.9 / 44^{\circ}$ & $2.9 / 320^{\circ}$ & $2.3 / 41^{\circ}$ & Eanes [2000] \\
3 & SLR/DORIS (T/P) & $2.1 / 62^{\circ}$ & $4.4 / 282^{\circ}$ & $4.0 / 105^{\circ}$ & Cretaux et al. [2002] \\
4 & SLR (Lageos 1 + 2) & $2.6 / 32^{\circ}$ & $2.5 / 309^{\circ}$ & $3.3 / 36^{\circ}$ & Cretaux et al. $[2002]$ \\
\hline
\end{tabular}

with the atmospheric mass [Flechtner, 2003]. Over the ocean, the combined effect of this atmosphere-ocean dealiasing (AOD) model is to estimate the non-IB barotropic signals. This is done to reduce high-frequency barotropic signals from aliasing into long-period errors in the GRACE gravity fields. It also means that the GRACE coefficients are actually variations relative to the monthly mean of the barotropic model. This is potentially a problem for studies involving a combination with altimetry if the mean barotropic signal is significant, since there is no barotropic model removed from the altimeter data. If the barotropic model is $100 \%$ correct on monthly timescales, then GRACE will observe the residual unmodeled signal (along with other mass variations), while the full barotropic variation will remain in the altimetry. On the other hand, if the model is $100 \%$ incorrect, then the GRACE coefficients have adjusted so that sum represents the full non-IB ocean variations, and so the GRACE data are biased by the error in the model. The truth undoubtedly lies between these extremes. Either way, adding the AOD model information back to the GRACE maps over the ocean will give maps of the full monthly non-IB barotropic and ocean mass variations. The time average of the AOD model used in the processing is available from PODAAC along with the gravity coefficients. Thus it is easy to map the modeled non-IB barotropic signal and add it back to the GRACE maps. Although the signal is small $(<1 \mathrm{~cm}$ RMS in general $)$ we do this to ensure consistency.

[33] There is one additional inconsistency that is more difficult to resolve. This is aliased high-frequency barotropic signals in the Jason 1 altimetry. Although this has been shown to be a problem in some areas and that using a model reduces the problem [Stammer et al., 2000; Tierney et al., 2000], no correction is available on the Jason 1 Geophysical Data Records (GDRs). This should be done to fully reconcile the GRACE and Jason 1 data, but is beyond the scope of this investigation. This means that differences in the regions noted by Stammer et al. [2000] and Tierney et al. [2000], generally the Southern Ocean, are possibly due to aliased signals in Jason 1, and not necessarily errors in GRACE. In this study, however, we will assume the differences are caused only by errors in GRACE. Thus, in regions of large high-frequency barotropic variability, our error estimates are likely overestimated.

\subsubsection{Summary of GRACE Processing}

[34] In short, the following processing steps are conducted on the GRACE data in order to combine them with altimetry. We have chosen this methodology because it is conducted solely on the GRACE products and it seems conceptually easier to the user than to mix some processing with procedures on altimetry (such as reprocessing Jason 1 data without correcting for the ocean pole tide).

[35] 1. Add degree 1 terms from a geocenter estimate (see section 3.1 and Table 1 for suggested models).

[36] 2. Substitute values for $\Delta C_{20}$ from an SLR analysis (after removing the atmosphere component from the AOD model) [e.g., Cox and Chao, 2002; Cheng and Tapley, 2004].

[37] 3. Map into equivalent water thickness averaged over a radius of $1000 \mathrm{~km}$ (equation (4)).

[38] 4. Add maps of non-IB barotropic SL from model used in GRACE processing.

[39] 5. Subtract average maps of ocean pole tide (averaged over same interval as GRACE coefficients).

[40] At this point, we have 21 maps of nonsteric SL from GRACE at approximately monthly intervals between August 2002 and July 2004 which have been as fully reconciled with altimetry SL as is currently possible. Then, given the smoothed SL maps from GRACE and altimetry, we calculate maps of ESSL as

$$
\operatorname{ESSL}(\phi, \lambda, t)=\Delta \eta_{\text {alt }}(\phi, \lambda, t)-\Delta \eta_{\text {mass }}(\phi, \lambda, t) .
$$

[41] Because of the gaps in the GRACE data, there are only 21 maps for the period starting in August 2002 and ending in July 2004. Because the ESSL and altimeter SL (ASL) maps will be compared to SSL from a climatology that has no interannual variability, we detrend the ESSL and ASL data. A bias and trend were fit to the 21 monthly maps along with annual and semiannual sinuosoids, then the bias and trend were removed.

\section{Discussion of Results}

\subsection{Effect of Geocenter Model}

[42] In order to quantify the importance of using a geocenter model for the GRACE data, we followed all the steps listed in section 2.4.5 except we made four test cases using different geocenter models as well as one case with no geocenter model (Table 1). The four geocenter models we examine represent tracking to two different types of satellites (LAGEOS 1 and 2 and T/P) and analysis by two different groups; the SLR model reported by Chen et al. [1999] was computed by R. Eanes. The models have similar values for $\Delta x$ and $\Delta y$, with standard deviations of less than a $\mathrm{mm}$ in amplitude and $15^{\circ}$ in phase. However, the difference in phase for the third $\Delta z$ solution is more than $69^{\circ}$ different from the closest model, while the other three agree to less than $11^{\circ}$. This highlights the greatest source of error in the geocenter solutions, that of determining the $\Delta z$ component, 
Table 2. Results of Variance Reduction Using Various Geocenter Models for GRACE ${ }^{\mathrm{a}}$

\begin{tabular}{lcccc}
\hline Model & $\begin{array}{c}\text { \% Area } \\
\text { Variance } \\
\text { Decreased }\end{array}$ & $\begin{array}{c}\text { \% Area } \\
\text { Variance } \\
\text { Increased }\end{array}$ & $\begin{array}{c}\text { Mean, RMS } \\
\text { Variance } \\
\text { Reduction }\end{array}$ & $\begin{array}{c}\text { Mean, RMS } \\
\text { Variance } \\
\text { Increase }\end{array}$ \\
\hline None & 50.7 & 49.3 & $1.0,1.7$ & $0.8,1.1$ \\
1 & 71.5 & 28.5 & $1.0,1.6$ & $0.9,1.2$ \\
2 & 74.3 & 25.7 & $1.1,1.7$ & $0.7,1.0$ \\
3 & 48.7 & 51.3 & $1.1,1.8$ & $1.3,1.2$ \\
4 & 69.4 & 30.6 & $1.1,1.7$ & $0.8,1.1$ \\
\hline
\end{tabular}

${ }^{\mathrm{a}}$ Geocenter model numbers are the same as in Table 1. Variance mean and RMS is in $\mathrm{cm}^{2}$.

which is related partly to the paucity of tracking stations in the Southern Hemisphere.

[43] We computed EOFs from the five ESSL cases (4 geocenter models + no model) as well as from the ASL and SSL grids. Although the ASL grids are complete for August 2002 to July 2004, we used only the 21 months corresponding to the GRACE data in the calculations. We used 21 SSL grids as well, substituting the mean month for the exact month (i.e., August $2002=$ August $2003=$ mean August for SSL).

[44] Before examining the recovered spatial patterns and time variations, we will examine the effect of the geocenter models. The goal of this process is to quantify how much improvement is found when a geocenter model is used with the GRACE data, and to determine which geocenter model is most consistent with the other data sets (i.e., leads to the smallest residuals). To do this, we compute reconstructed grids $\left(h^{R}\right)$ based on the leading EOF mode (equation (1))

$$
h^{R}(\phi, \lambda, t)=\alpha_{1}(\phi, \lambda) \beta_{1}(t),
$$

for the ESSL, ASL, and SSL grids, and then compute the variance of the residuals for ASL and ESSL reconstructed grids relative to the SSL reconstructed grids

$$
\begin{aligned}
\operatorname{var}[\operatorname{ASL}(\phi, \lambda)] & =\frac{1}{21} \sum_{t=1}^{21}\left[\operatorname{ASL}^{R}(\phi, \lambda, t)-\operatorname{SSL}^{R}(\phi, \lambda, t)\right]^{2} \\
\operatorname{var}[\operatorname{ESSL}(\phi, \lambda)] & =\frac{1}{21} \sum_{t=1}^{21}\left[\operatorname{ESSL}^{R}(\phi, \lambda, t)-\operatorname{SSL}^{R}(\phi, \lambda, t)\right]^{2}
\end{aligned}
$$

We then computed the percentage of area where $\operatorname{var}[E S S L]$ had a lower value than $\operatorname{var}[A S L]$, as well as the average and RMS values over that area (Table 2). This indicates where using the GRACE information improved the results over using ASL alone. We also computed the percentage of grids where the variance increased, meaning that using GRACE was not as good as using altimetry alone. If no geocenter model is used, the percentage of area with a reduced variance is about equal to that where the variance increased. However, when a geocenter model is utilized, the variance is typically reduced over more than $70 \%$ of the ocean. The one exception is when the third geocenter model (which stands out with a very different $\Delta z$ from the other models) is used. It actually performs worse than if no geocenter model is used. This suggests that using a bad geocenter estimate can do more harm than just ignoring the signal altogether in this type of analysis. However, the results also suggest that a good geocenter estimate (based on consistency with other estimates) can improve the results compared with ignoring the signal.

[45] We note also that in the areas where the variance has decreased, the change is larger than in the areas where the variance has increased. For example, using the model computed from the Eanes [2000] time series (model 2), the mean decrease in variance of $1.1 \mathrm{~cm}^{2}$, with an RMS of $1.7 \mathrm{~cm}^{2}$, calculated over the $74 \%$ of the ocean where the variance decreased. In the $26 \%$ of the ocean where the variance increased, the mean increase was $0.7 \mathrm{~cm}^{2}$, and an RMS of $1.0 \mathrm{~cm}^{2}$. This means that on average, the decrease in variance was more than the increase, and that the maximum decrease was larger than the maximum increase. Because the Eanes [2000] model performed best in our tests, we use it for the remainder of the analysis, although we will show several plots where no geocenter is used, just to highlight the difference.

\subsection{Leading EOF Mode}

[46] Figure 5 shows the spatial map of the leading EOF mode for the WOA01 SSL, Jason 1 SL (ASL) with no GRACE correction, and ESSL from the combination of

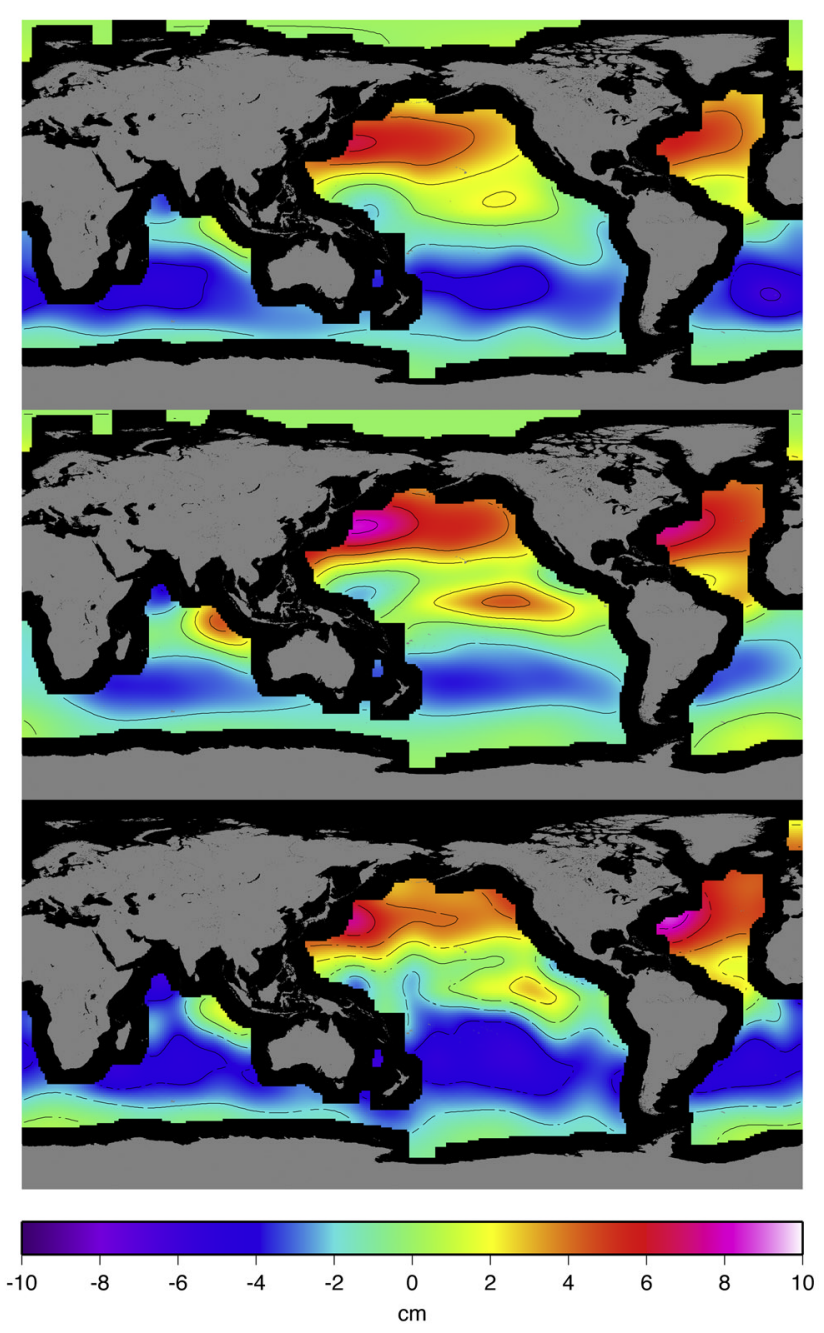

Figure 5. Leading spatial EOF mode for (top) SSL, (middle) ASL, and (bottom) ESSL. Mode has been scaled to represent maximum amplitude. 


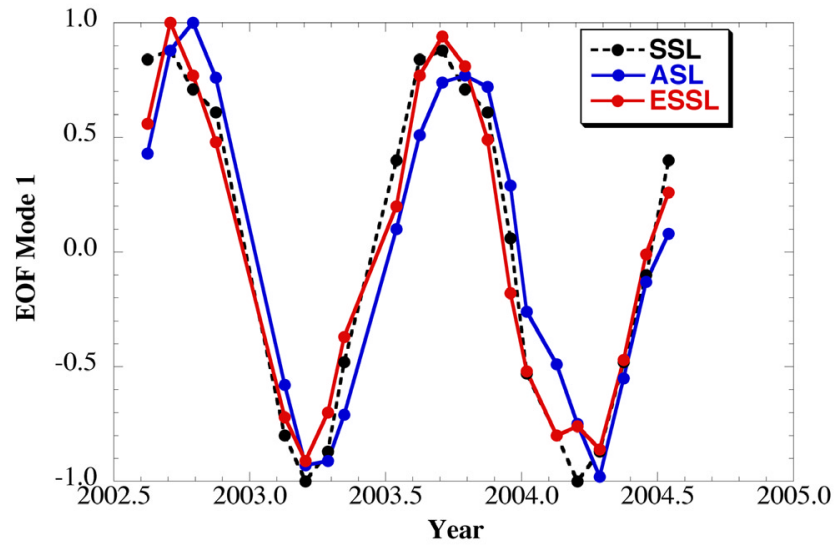

Figure 6. Time series of EOF Mode 1, scaled so that reconstructed maps are calculated when multiplied by pattern in Figure 5.

GRACE and Jason 1 (with the geocenter correction). Figure 6 shows the associated time series (principal components). The leading EOF mode explains $76 \%$ of the SSL variance, $68 \%$ of ASL, and $52 \%$ of ESSL. The periodicity of the leading mode is similar for all data with a dominant annual cycle, but the peak in ASL is one month later than that of SSL. However, when GRACE data are added to compute ESSL, the phase is shifted by a month so that ESSL is in slightly better agreement with that of the SSL.

[47] Although the patterns of the leading SSL and ASL modes are generally similar, there are noticeable differences. The ASL pattern has a higher peak in the Northern Hemisphere $(\mathrm{NH})$ compared to SSL, whereas the opposite is true in the Southern Hemisphere $(\mathrm{SH})$. The reason for this can be partly explained by the timing of water mass storage over the ocean as part of the global water cycle. Chambers et al. [2004] found that more water mass was stored in the ocean, on average, in early October of each year, with a subsequent minimum 6 months later. The amplitude is $8-$ $9 \mathrm{~mm}$ of SL. Theoretically, the ocean SL should readjust to water mass fluxes via barotropic waves and distribute the signal more or less uniformly over the oceans within a few days. Assuming this is true, then more water mass is stored in the ocean at approximately the same time of the year as the maximum SSL in the NH, which would cause total sea level measured by Jason 1 to be larger. In the $\mathrm{SH}$, the timing of water mass storage is nearly $180^{\circ}$ out of phase with maximum SSL, so there is a reduction in the amplitude of total SL. This can explain part of the difference, but not all of it. For example, the ASL also has higher amplitudes than SSL in portions of the tropical Indian and Pacific Oceans, but agree to less than a few $\mathrm{mm}$ in other areas. This suggests that local effects from mass redistribution have an influence as well.

[48] The pattern for ESSL is closer to the pattern of SSL. The NH and SH patterns are more similar, and the large amplitudes in the tropical Indian and Pacific Oceans have been reduced by up to $2 \mathrm{~cm}$. The RMS difference between the ASL and SSL maps is $1.3 \mathrm{~cm}$ and the correlation is 0.89 , while the RMS difference between the SSL and ESSL patterns has been reduced to $0.9 \mathrm{~cm}$, and the correlation increased to 0.95 . The seasonal difference between ASL and
SSL in the eastern tropical Pacific was briefly noted in an earlier study [Chambers et al., 1998], with no explanation. These results however suggest that the cause is a variation in mass.

[49] The statistics comparing the EOF spatial modes do not give the whole picture, though, since there are some phase differences in the principal components. Examining the reconstructed grids (equation (12)) provides more information. The variance of the ASL and ESSL residuals relative to SSL (equation (13)) are displayed in Figure 7. The variance computed from ESSL without a geocenter correction is also displayed. If the GRACE measurements have improved the determination of SSL, then the ESSL variance should be lower than the ASL variance (provided there are no correlated errors which cause cancellation); if not, the variance should be higher. We have also computed the total variance on the basis of all the residuals. The total variance for the ASL residuals is $1.1 \mathrm{~cm}^{2}$. The variance of the ESSL residuals is about half of this if the geocenter
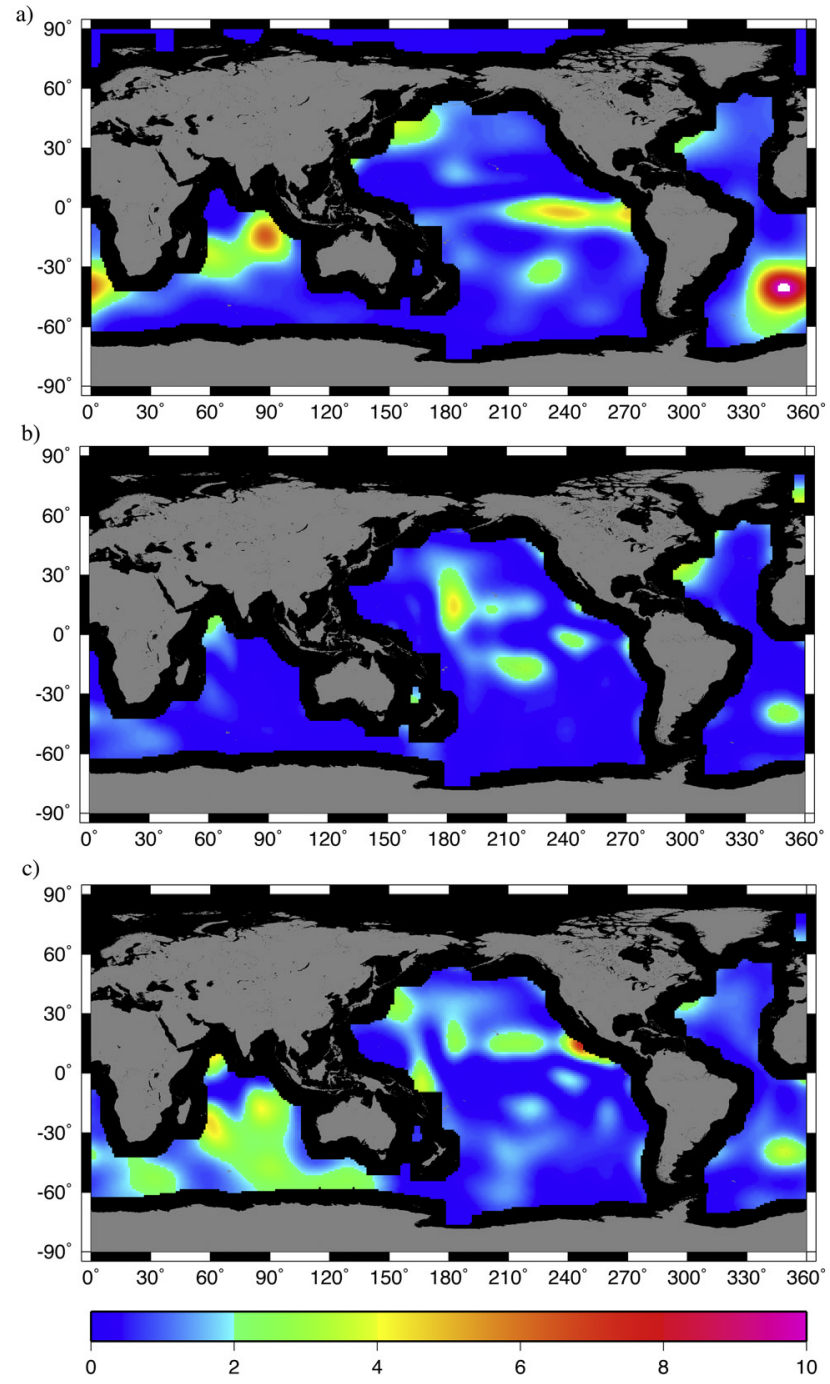

Figure 7. Variance of EOF1 reconstructed residuals (in $\mathrm{cm}^{2}$ ): (a) ASL-SSL, (b) ESSL (with geocenter) - SSL, and (c) ESSL (without geocenter) - SSL. 
a)

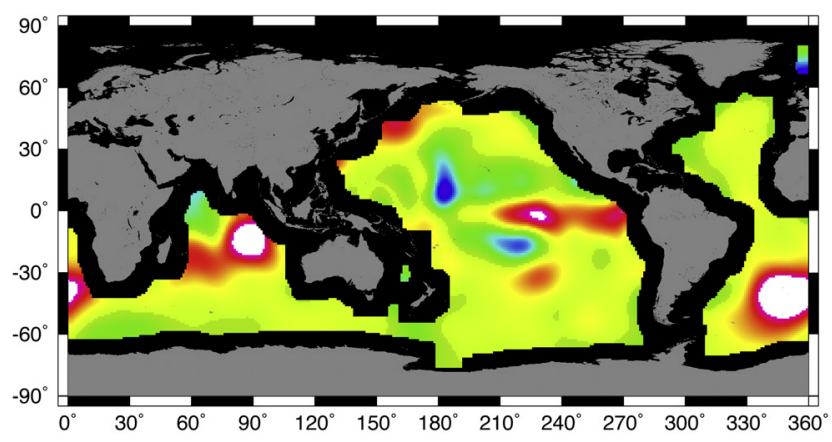

b)

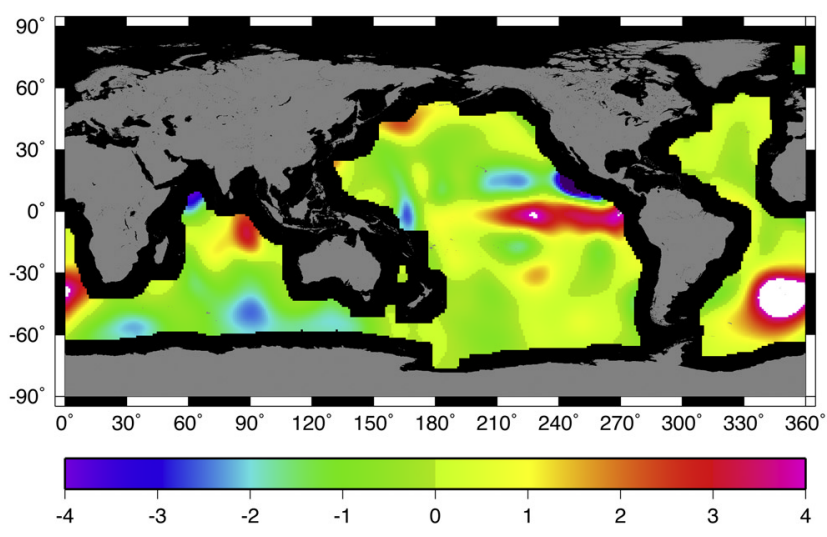

Figure 8. Variance reduction for EOF1 reconstructed ESSL compared to reconstructed ASL (in $\mathrm{cm}^{2}$ ) for (a) GRACE with geocenter and (b) GRACE without geocenter.

model is used $\left(0.6 \mathrm{~cm}^{2}\right)$ and comparable if the geocenter model is not used $\left(1.1 \mathrm{~cm}^{2}\right)$.

[50] The largest changes are in the tropical Indian Ocean, the eastern tropical Pacific, the South Atlantic, and South of Australia. So see this more clearly, we compute reduction in variance, or

$$
-(\operatorname{var}[E S S L]-\operatorname{var}[A S L])
$$

where the negative sign is used so that a reduction in $\operatorname{var}[E S S L]$ is positive, while an increase is negative. Results are shown in Figure 8. The variance reduction is as much as $8 \mathrm{~cm}^{2}$ in the South Atlantic, and, as noted earlier in the evaluation of the geocenter models (Table 2), the variance is reduced over nearly three quarters of the ocean. This suggests that GRACE is contributing significant information to improve estimation of the dominant annual variation in SSL over much of the ocean.

[51] The one notable exception is a broad region in the Pacific Ocean around $180^{\circ} \mathrm{E}$. Here, the variance using GRACE is more than $4 \mathrm{~cm}^{2}$ higher than using altimetry alone. It is interesting to note that although not using a geocenter correction does not reduce the ESSL variance over many portions of the ocean as much as using a correction (Figure 8b), it also does not increase the variance significantly in this region of the Pacific. This suggests that the problem may lie in the geocenter correction, in partic- ular the $\Delta x$ component which lies along the line from $180^{\circ} \mathrm{E}$ to $0^{\circ} \mathrm{E}$ through the equatorial plane. Recall that the gravitational variation has a wavelength of $40000 \mathrm{~km}$, and so will spread the signal out considerably as will its interaction with errors in the other two components. However, the fact that all the models have a similar $\Delta x$ value would mean that all the models are wrong by the same amount. One way that this could happen is due to the tracking station distribution. There are many SLR tracking stations in western Europe (near $0^{\circ}$ longitude), but only two in the central Pacific. Since the geocenter estimate is based on estimating parameters to minimize SLR residuals, it is possible the $\Delta x$ solutions are biased toward the European sites.

[52] On the other hand, the signal could also be caused by an error in the GRACE data that is unconnected to the geocenter model at all. We find some evidence for this when we examine the second EOF mode.

\subsection{Second EOF Mode}

[53] Figure 9 shows the spatial map of the second EOF mode for the SSL, ASL, and ESSL, while Figure 10 shows

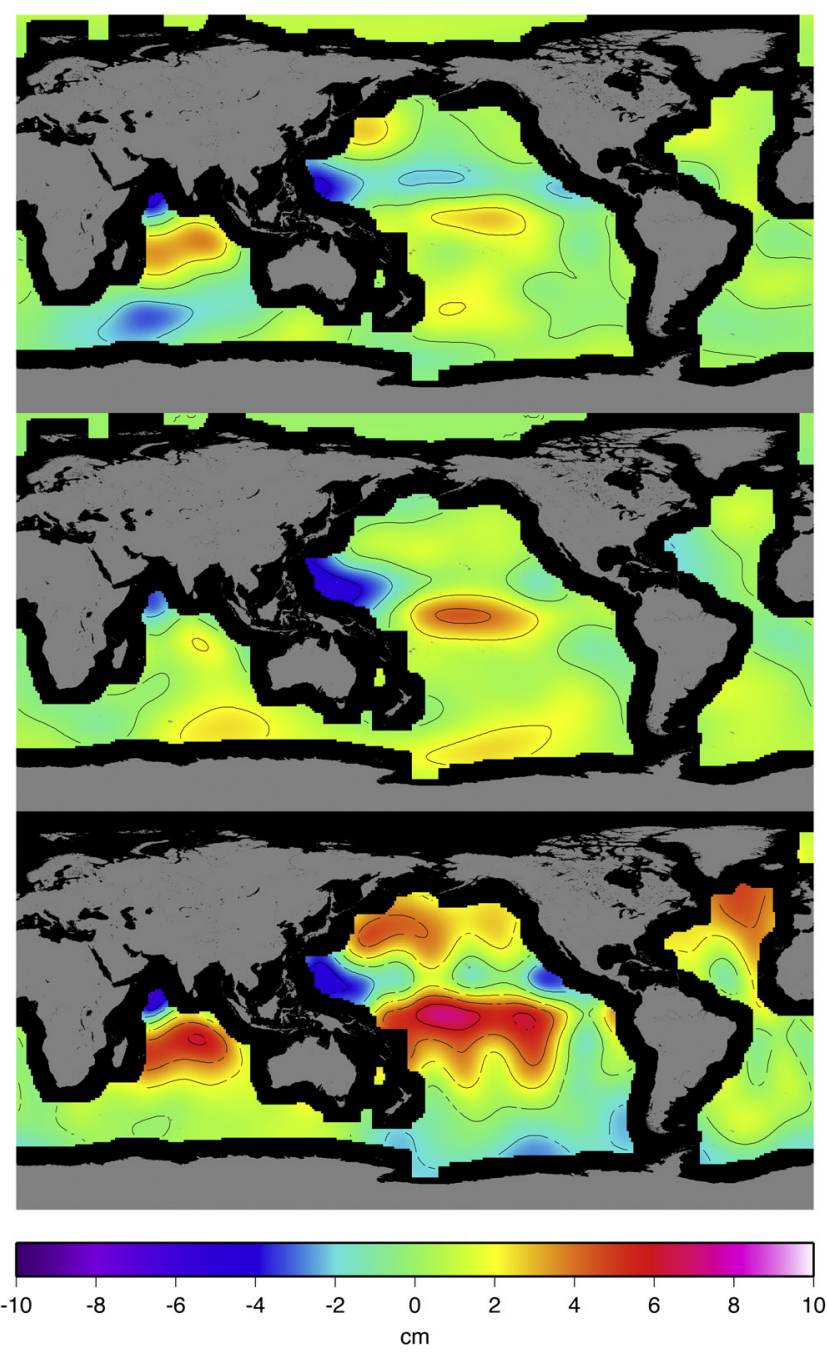

Figure 9. Second spatial EOF mode for (top) SSL, (middle) ASL, and (bottom) ESSL. Mode has been scaled to represent maximum amplitude. 


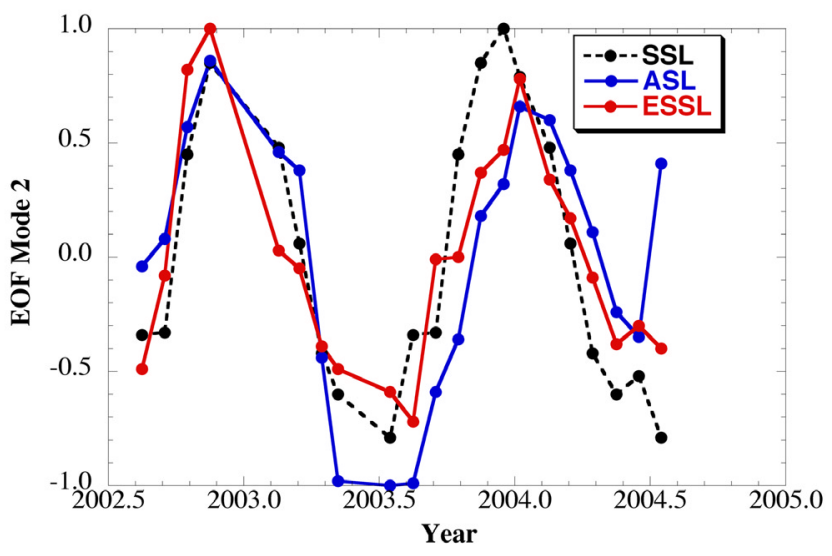

Figure 10. Time series of EOF Mode 2.

the associated time series. The second EOF mode explains $9 \%$ of the SSL variance, $8.5 \%$ of ASL, and $16 \%$ of ESSL. The periodicity is primarily annual like the first mode, but occurs approximately 90 days later. The RMS difference between the ASL and SSL maps is $1.4 \mathrm{~cm}$ and the correlation is 0.40 . The RMS difference between the SSL and ESSL patterns is $2.1 \mathrm{~cm}$, although the correlation increases to 0.57. From comparing the maps in Figure 9, it appears that adding GRACE data leads to a better determination of the patterns of variability compared with using Jason 1 alone, although the amplitudes are larger by as much as $2-4 \mathrm{~cm}$. Note the similar patterns in the North Pacific east of Japan, in the Indian Ocean, and in the eastern Pacific for the ESSL and SSL maps that are not as clearly defined in the ASL maps. One thing that could cause such a pattern would be a periodic error in the GRACE data that is nearly in phase with this SSL mode.

[54] Compare the ESSL EOF2 pattern in Figure 9 with the EOF1 pattern from the GRACE maps alone (Figure 3c) The largest deviations are in the same general locations; in fact, the correlation between the two maps is 0.71 . The timing of the maximum deviations is nearly $180^{\circ}$ out of phase (Figure 4 compared with Figure 10). The maximum positive GRACE SL variation for mode 1 is at the time of the maximum negative SSL variation in mode 2 . Thus it appears there is a significant error in the GRACE data at these locations that is correlated with some real variability, since it is included in the leading EOF mode.

[55] It is not clear what causes the error in the GRACE data that correlates with the SSL second EOF mode. In addition to the geocenter correction discussed previously, it could be a problem in the estimation of the gravity coefficients from the raw tracking data, or errors in the background models used in the processing (such as the ocean tide model). The only way to test this would be to completely reprocess the GRACE data with different models and see if any of them fix the problem. Several changes are being done in the GRACE processing, from changing the reference mean gravity field to using a higher-resolution ocean tide model and adding an ocean pole tide model (S. Bettadpur, personal communication, 2005). We will have to wait until new gravity fields are released in order to evaluate if these steps have reduced the error.
[56] However, even though there is apparently an error in the GRACE data that currently affects the recovery of ESSL in the tropical band beyond the leading EOF mode (especially in the western Pacific), we do note that results for much of the SH do continue to look better if GRACE is used than when it is not. We have computed reconstructed grids on the basis of the first two modes, and computed the variance reduction in ESSL compared to using ASL as before (Figure 11). The variance has been reduced further in several places in the $\mathrm{SH}$ when the two modes are used compared to just using the first mode.

\subsection{GRACE Signal-to-Noise Ratio}

[57] Although the previous results suggest GRACE is observing some real ocean mass variations, they are dependent on using EOFs, which are a special space-time filter. What is the error in the GRACE observations if EOF filtering is not used? One can quantify this to a certain extent if it is assumed that the difference in the ESSL - SSL residuals is due solely to GRACE errors. This ignores any contribution from errors in the altimetry (such as the aliasing of high-frequency barotropic SL) or the SSL data, as well as correlated GRACE errors. The RMS of the residuals where no EOF reconstruction has been applied is shown in Figure 12. The mean RMS is $2.3 \mathrm{~cm}$, with the largest differences occurring in the tropical Pacific. Here the RMS is as large as $4 \mathrm{~cm}$.

[58] This suggests the errors in the GRACE ocean mass variations are on average about $2 \mathrm{~cm}$ RMS. However, it is just as important to know where the expected signal is larger than this error. Consider that the variance of the ASL - SSL residuals represent one estimate of the power of the ocean mass variations. On the basis of this, one can define a signal-to-noise ratio (SNR) for the GRACE data as

$$
S N R(\phi, \lambda)=\frac{\sum_{t=1}^{21}\left[\Delta \eta_{\text {alt }}(\phi, \lambda, t)-\Delta \eta_{S S L}(\phi, \lambda, t)\right]^{2}}{\sum_{t=1}^{21}\left[\left(\Delta \eta_{\text {alt }}(\phi, \lambda, t)-\Delta \eta_{\text {mass }}(\phi, \lambda, t)\right)-\Delta \eta_{S S L}(\phi, \lambda, t)\right]^{2}} .
$$

Figure 13 shows the estimated GRACE SNR. The ratio is greater than 2 in several places, most significantly in the

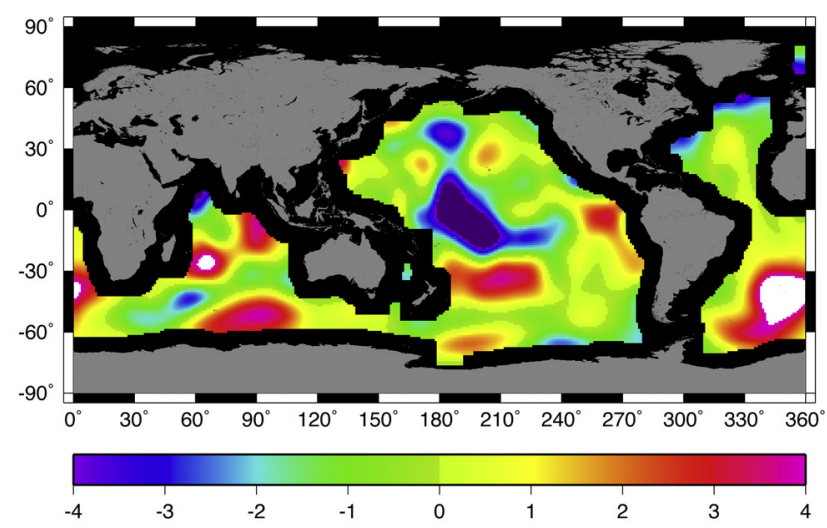

Figure 11. Variance reduction for EOF1-2 reconstructed ESSL compared to reconstructed ASL (in $\mathrm{cm}^{2}$ ). 
South Indian Ocean, the South Pacific (around $30^{\circ} \mathrm{S}$ and $60^{\circ} \mathrm{S}$ ) and in the South Atlantic, suggesting a benefit from the GRACE data is possible.

\section{Conclusions}

[59] There has been a notion among many investigators that the time variable gravity signal currently recovered from GRACE is not accurate enough to observe mass variability over the ocean. Part of this is based on comparison to ocean models that do not account for the hydrological cycle [e.g., Wahr et al., 2004], which is not insignificant at the $1 \mathrm{~cm}$ level. We evaluated GRACE data in a different fashion, by combining it with Jason 1 altimetry (which does observe the global water cycle) and comparing the estimated steric sea level variations with the seasonal variations derived from the World Ocean Atlas. We discussed several important corrections that currently need to be made in order to reconcile the GRACE data with Jason 1. We also used EOF analysis in order to examine out of phase seasonal variations more closely.

[60] On the basis of our studies, we conclude that GRACE is observing real ocean mass variations that can be combined with altimetry to estimate SSL in several regions of the ocean. For the dominant annual SSL variation, using GRACE improves the estimation over using altimetry alone for about $75 \%$ of the ocean area. The major exception is in the western Pacific. The largest improvements occur in the Indian Ocean, the eastern Pacific, and the South Atlantic. The second mode of SSL variation is also annual, but it appears that GRACE may have time variable errors correlated with the mode that affect the tropical region in particular. However, results in the Southern Hemisphere continue to improve when GRACE is used.

[61] It is not clear from this analysis what causes the error in the GRACE data that correlates with the SSL second EOF mode. It could be a problem converting the raw GRACE measurements to gravity coefficients, or errors in the background models used in the processing. Several changes are being done in the processing, from changing the reference mean gravity field to adding a higher-resolution ocean tide model and adding an ocean pole tide model

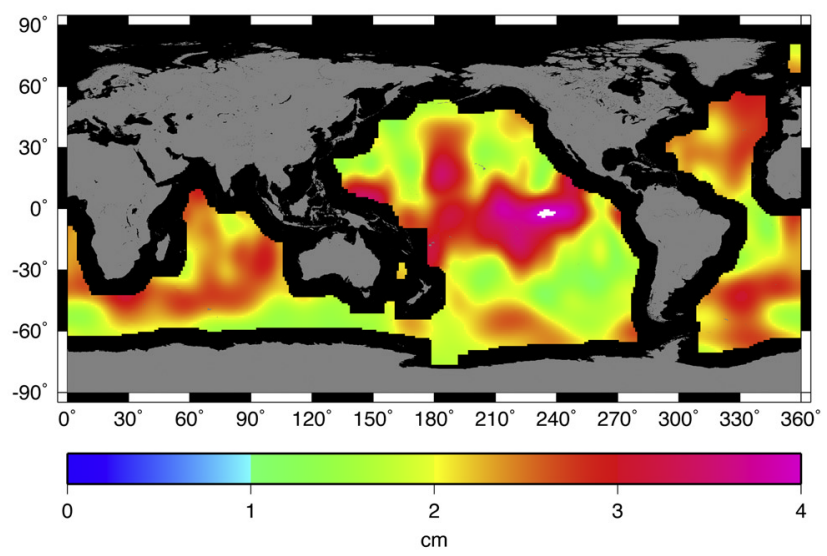

Figure 12. Estimated RMS error for ocean mass variations from GRACE, based on residuals of ESSL-SSL, assuming all of difference is due to GRACE errors.

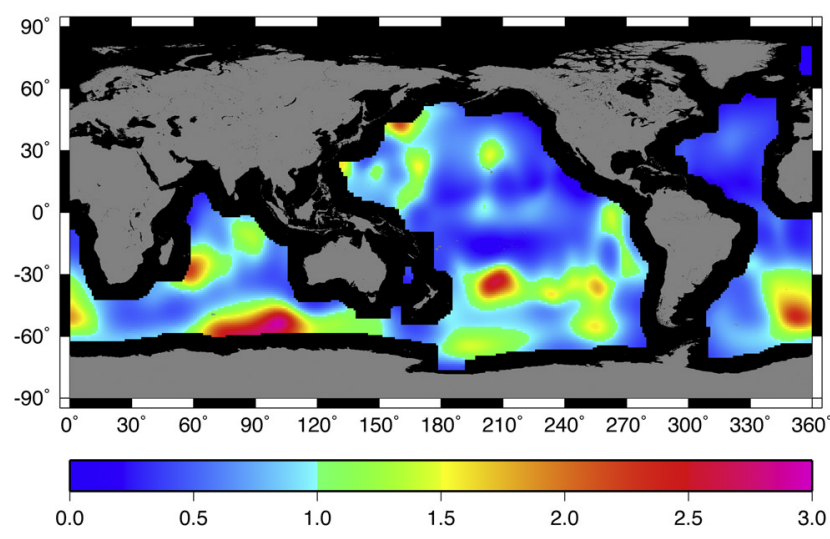

Figure 13. Estimated signal-to-noise ratio for ocean mass variations from GRACE.

(S. Bettadpur, personal communication, 2005). We will have to wait until new gravity fields are released in order to evaluate if these steps have reduced the error. It could also be errors in either the geocenter model correction (especially the $\Delta x$ component), or the $\Delta C_{20}$ substitution we have made, or a combination of the two. The latter problem may be resolved in the reprocessing based on early results as newer GRACE estimates of $\Delta C_{20}$ appear to be more realistic (S. Bettadpur, personal communication, 2005).

[62] It does appear, though, that even with these problems, GRACE errors are smaller than the expected signal in many places. We have estimated that the RMS error of the GRACE data is equivalent to $2-3 \mathrm{~cm}$ for $1000 \mathrm{~km}$ smoothing with no EOF filtering over most of the ocean. Errors are higher in the western tropical Pacific. This is more than likely a higher limit, since it assumes that the altimeter and SSL data are error free. Although this error is larger than initially predicted for GRACE $(<1 \mathrm{~cm}$ for this smoothing radius), it is still smaller than the expected signal for many regions (Figure 13). Thus we believe that GRACE can provide useful information in some regions of the ocean, and hope that other investigators may begin to use the data in order to gain more insight into the dynamics of sea level variability.

[63] Acknowledgments. TOPEX, Jason 1, and GRACE data are from Physical Oceanography Data Archive Center at Jet Propulsion Laboratory/ California Institute of Technology. The WOA01 data are from the National Oceanographic Data Center. We would like to thank M. Cheng for providing his $\mathrm{C}_{20}$ time series. We are also grateful to the three reviewers who made many suggestions to improve this paper. This research was supported by the NASA Solid Earth Natural Hazards program under grant NNG04GF11G and Jet Propulsion Laboratory under contract 1226830 .

\section{References}

Bettadpur, S. (2004), Level-2 gravity field product user handbook, GRACE 327-734, 17 pp., Univ. of Tex. at Austin, Austin.

Chambers, D. P. (2002), Effect of sea level variability on the estimation of mean sea surface gradients, Mar. Geod., 25, 273-288.

Chambers, D. P., B. D. Tapley, and R. H. Stewart (1998), Measuring heatstorage changes in the equatorial Pacific: A comparison between TOPEX altimetry and TAO buoys, J. Geophys. Res., 103, 18,591-18,597.

Chambers, D. P., J. Wahr, and R. S. Nerem (2004), Preliminary observations of global ocean mass variations with GRACE, Geophys. Res. Lett., 31, L13310, doi:10.1029/2004GL020461. 
Chao, B. F., and R. J. Eanes (1995), Global gravitational change due to atmospheric mass redistribution as observed in Lageos' nodal residual, Geophys. J. Int., 122, 755-764.

Chen, J. L., C. R. Wilson, D. P. Chambers, R. S. Nerem, and B. D. Tapley (1998), Seasonal global water mass balance and mean sea level variations, Geophys. Res. Lett., 25, 3555-3558.

Chen, J. L., C. R. Wilson, R. J. Eanes, and R. S. Nerem (1999), Geophysical interpretation of observed geocenter variations, J. Geophys. Res., 104, 2683-2690.

Cheng, M., and B. D. Tapley (2004), Variations in the Earth's oblateness during the past 28 years, J. Geophys. Res., 109, B09402, doi:10.1029/ 2004JB003028.

Cox, C. M., and B. F. Chao (2002), Detection of a large-scale mass redistribution in the terrestrial system since 1998, Science, 297, 831-833.

Cretaux, J.-F., L. Soudarin, F. J. M. Davidson, M.-C. Gennero, M. BergeNguyen, and A. Cazenave (2002), Seasonal and interannual geocenter motion from SLR and DORIS measurements: Comparison with surface loading data, J. Geophys. Res., 107(B12), 2374, doi:10.1029/ 2002JB001820

Eanes, R. (2000), SLR Solutions From the University of Texas Center for Space Research, Geocenter from TOPEX SLR/DORIS, 1992-2000, http://sbgg.jpl.nasa.gov/datasets.html, IERS Spec. Bur. for Gravity/Geocent., Pasadena, Calif.

Flechtner, F. (2003), AOD1B product description document, GRACE 327750, 35 pp., Univ. of Tex. at Austin, Austin.

Fu, L. L. (Ed.) (2004), Minutes of the Ocean Surface Topography Science Team Meeting, JPL Rep. D-31211, pp. 16-17, Jet Propul. Lab., Pasadena, Calif.

Heiskanen, W. A., and H. Moritz (1967), Physical Geodesy, W. H. Freeman, New York.

Hughes, C. W., and M. J. Smithson (1996), Bottom pressure correlations in the south Atlantic, Geophys. Res. Lett., 23, 2243-2246.

Jayne, S. R., J. M. Wahr, and F. O. Bryan (2003), Observing ocean heat content using satellite gravity and altimetry, J. Geophys. Res., 108(C2), 3031, doi:10.1029/2002JC001619.

Levitus, S., C. Stephens, J. Antonov, and T. Boyer (2000), Yearly and year-season upper ocean temperature anomaly fields, 1948-1998, NOAA Atlas NESDIS 40, Natl. Oceanic and Atmos. Admin., Silver Spring, Md.

Menkes, C., J.-P. Boulanger, and A. J. Busalacchi (1995), Evaluation of TOPEX and basin-wide Tropical Ocean and Global Atmosphere-Tropical
Atmosphere Ocean sea surface topographies and derived geostrophic currents, J. Geophys. Res., 100, 25,087-25,099.

Minster, J. F., A. Cazenave, and P. Rogel (1999), Annual cycle in mean sea level from TOPEX-Poseidon and ERS-1: Inference on the global hydrological cycle, Global Planet. Change, 20, 57-66.

Nerem, R. S., R. J. Eanes, P. Thompson, and J. L. Chen (2000), Observations of seasonal variations of the Earth's gravity field using satellite laser ranging and geophysical models, Geophys. Res. Lett., 27, 1783-1786.

Ponte, R. (1999), A preliminary model study of the large-scale seasonal cycle in bottom pressure over the global ocean, J. Geophys. Res., 104, $1289-1300$

Preisendorfer, R. W. (1988), Principal Component Analysis in Meteorology and Oceanography, edited by C. Mobley, 418 pp., Elsevier Sci., New York.

Stammer, D., C. Wunsch, and R. M. Ponte (2000), De-aliasing of global high frequency barotropic motions in altimeter measurements, Geophys. Res. Lett., 27, 1175-1178.

Stephens, J. I., T. P. Antonov, T. P. Boyer, M. E. Conkright, R. A. Locarnini, T. D. O'Brien, and H. E. Garcia (2002), World Ocean Atlas 2001, vol. 1, Temperatures, edited by S. Levitus, NOAA Atlas NESDIS 49, 176 pp., U. S. Gov. Print. Off., Washington, D. C.

Tierney, C., J. Wahr, F. Bryan, and V. Zlotniki (2000), Short-period oceanic circulation: Implications for satellite altimetry, Geophys. Res. Lett., 27, $1255-1258$

Wahr, J. (1985), Deformation induced by polar motion, J. Geophys. Res., 90, 9363-9368.

Wahr, J., M. Molenaar, and F. Bryan (1998), Time-variability of the Earth's gravity field: Hydrological and oceanic effects and their possible detection using GRACE, J. Geophys. Res., 103, 32,205-30,229.

Wahr, J., S. Swenson, V. Zlotnicki, and I. Velicogna (2004), Time-variable gravity from GRACE: First results, Geophys. Res. Lett., 31, L11501, doi:10.1029/2004GL019779.

Willis, J. K., D. Roemmich, and B. Cornuelle (2004), Interannual variability in upper ocean heat content, temperature, and thermosteric expansion on global scales, J. Geophys. Res., 109, C12036, doi:10.1029/ 2003JC002260.

D. P. Chambers, Center for Space Research, University of Texas at Austin, 3925 West Braker Lane, Ste. 200, Austin, TX 78759-7483, USA. (chambers@csr.utexas.edu) 